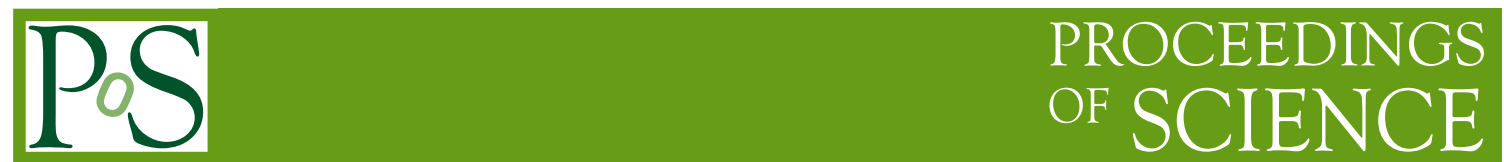

\title{
Analyticity constrained pion-nucleon analysis
}

\section{M.E. Sainio}

Helsinki Institute of Physics, P.O. Box 64, 00014 University of Helsinki, Finland

E-mail: mikko.sainio@helsinki.fi

\begin{abstract}
A brief progress report is given on the partial wave analysis of pion-nucleon scattering. Fixed- $t$ constraints are implemented with the expansion techniques and for the forward amplitudes with accurate data a solution is found. Extending the analysis away from the forward direction will require a careful data amalgamation.
\end{abstract}

6th International Workshop on Chiral Dynamics

July 6-10 2009

Bern, Switzerland 


\section{Introduction}

The pion-nucleon scattering amplitude can be presented in terms of the invariant amplitudes $A$ and $B$ as, see e.g. Ref.[1],

$$
T_{\pi N}=\bar{u}^{\prime}\left[A(v, t)+\frac{1}{2} \gamma^{\mu}\left(q+q^{\prime}\right)_{\mu} B(v, t)\right] u
$$

where

$$
v=\frac{s-u}{4 m}=\omega+\frac{t}{4 m} .
$$

The proton mass is denoted by $m$ and $\omega$ is the pion total energy in the laboratory system. Instead of $A$ and $B$ it is convenient to use $C$ and $B$, where the amplitude $C$ is defined as

$$
C(v, t)=A(v, t)+\frac{v}{\left(1-t / 4 m^{2}\right)} B(v, t) .
$$

Through the optical theorem the imaginary part of the $C$-amplitude at $t=0$ is related to the total cross section in the corresponding physical channel

$$
\operatorname{Im} C(\omega, t=0)=k_{\mathrm{lab}} \sigma
$$

and this is exploited in the study of the forward dispersion relations for $C$. The isoscalar and isovector amplitudes are defined as

$$
C^{ \pm}=\frac{1}{2}\left(C_{\pi^{-} p} \pm C_{\pi^{+} p}\right)
$$

The amplitudes $A$ and $B$ satisfy fixed- $t$ dispersion relations, see e.g. Ref. [1], and they can be proven rigorously $[2,3]$ for a range of $t$-values

$$
4 \mu^{2} \geq t \geq-(2 m+\mu)\left[\frac{3 \mu(2 m+3 \mu)}{(m+2 \mu)^{2}}+\frac{4 \mu}{m}\right] \mu \simeq-18 \mu^{2} \simeq-0.35 \mathrm{GeV}^{2}
$$

by employing microscopic causality and unitarity. Here the pion mass is denoted by $\mu$. It has also been shown $[4,5]$ that the underlying confining gauge theory, QCD, does not change the outcome, the proofs for the fixed- $t$ dispersion relations remain valid.

The basic requirements for a pion-nucleon analysis are analyticity, crossing and unitarity. The fixed- $t$ dispersion relations are conventionally written in a form which guarantees the crossing property.

Isospin is an approximate symmetry and it is broken in pion-nucleon scattering by the electromagnetic interaction and the quark mass difference $m_{u} \neq m_{d}$. In the present analysis the aim is to work with amplitudes which satisfy isospin symmetry. This is achieved by removing from the experimental data the electromagnetic effects by using the Tromborg formalism [6]. At the lowest energies the question may arise of the validity of the Tromborg approach in estimating the electromagnetic corrections [7]. However, for the present analysis there is no practical alternative, because we make use of the corrections up to $655 \mathrm{MeV} / \mathrm{c}$, the highest momentum point available in the Nordita analysis [6]. The range of validity of the third order chiral perturbation theory calculation [7] is not known and, in any case, the weight of the experimental data below the laboratory 


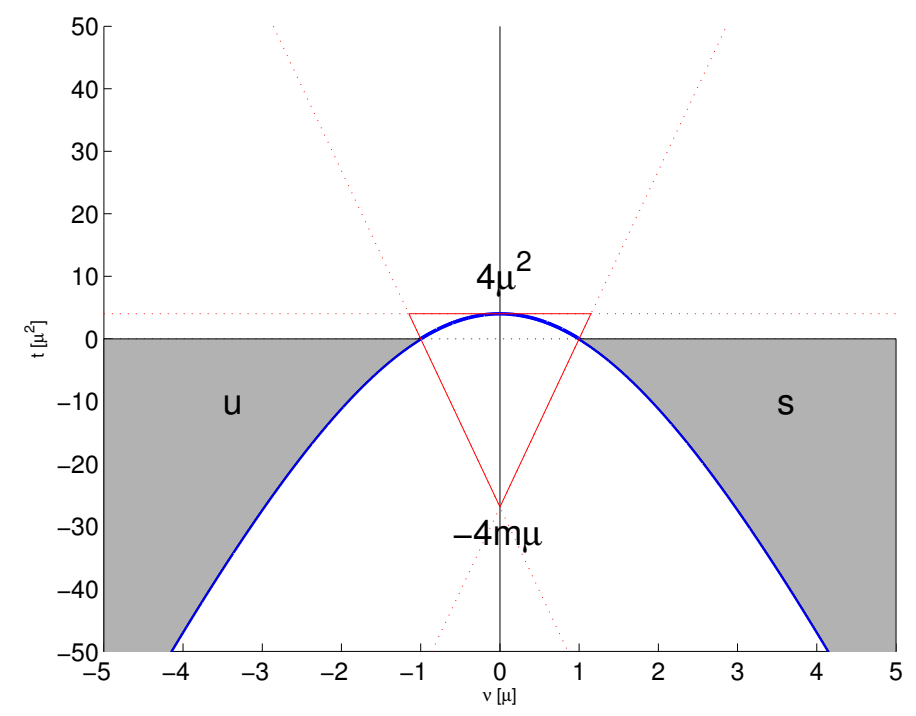

Figure 1: The Mandelstam diagram for $\pi \mathrm{N}$ scattering.

momentum $100 \mathrm{MeV} / \mathrm{c}$ is small in an analysis extending to highest energies. The $\Delta$-splitting effect was taken into account by using the $\mathrm{P}_{33}$ phase shift differences of Ref.[8] which are quite similar to the figures of Bugg [9]. After applying these corrections to the data it is assumed that the remaining isospin violating effects are small in comparison with the experimental uncertainties.

Constraints from fixed- $t$ dispersion relations and isospin invariance are then sufficient for resolving the ambiguities of phase-shift analysis [1].

\section{The expansion techniques}

Fixed- $t$ dispersion relations have a particular feature due to the $s-u$ crossing, there are no unknown left-hand cuts [1], see Fig.1. For the range $0 \geq t \geq-4 m \mu \simeq-0.52 \mathrm{GeV}^{2}$ the $s$ - and the $u$-channel cuts do not overlap. There is, however, the unphysical region between the Mandelstam triangle $(v= \pm \mu \pm t / 4 m)$ and the physical area (the shaded area) for each line $t=$ constant. In these sections the partial wave expansions for the absortive parts of the invariant ampltitudes $\mathrm{A}$ and $\mathrm{B}$ converge for $0 \geq t \geq-0.5 \mathrm{GeV}^{2}[1]$ and that provides a means to evaluate the dispersion relations over the whole range of $v$-values. A rigorous proof of convergence for the imaginary parts of the the partial wave expansions exists for the range of $t$-values given in Eq.(1.4). Dispersion relations for $C^{ \pm}$can be obtained by combining those for $A^{ \pm}$and $B^{ \pm}$.

In the case of forward scattering $(t=0)$ it could still be considered feasible to evaluate dispersion integrals, but the extension to $t \neq 0$ would be difficult without adopting the expansion method where the invariant amplitude is expressed as a series of analytic functions. Here we employ a particular form for the expansion, the Pietarinen's expansion [1, 10, 11]. For example, for the $C^{+}$-amplitude one has

$$
C^{+}(v, t)=C_{N}^{+}(v, t)+H(Z, t) \sum_{n=0}^{N} c_{n}^{+} Z^{n}
$$


where $C_{N}^{+}(v, t)$ is the pole term, $\mathrm{H}$ is adjusted to the asymptotic behaviour of the amplitude and

$$
Z\left(v^{2}, t\right)=\frac{\alpha-\sqrt{v_{t h}^{2}-v^{2}}}{\alpha+\sqrt{v_{t h}^{2}-v^{2}}},
$$

with $\alpha=0.72 \mathrm{GeV}$ and $v_{t h}=\mu+t / 4 m$. This maps the physical region on the upper semicircle of the unit circle.

The coefficients $c_{n}^{+}$in the expansion can be determined by minimizing

$$
\chi^{2}=\chi_{D A T A}^{2}+\chi_{P W}^{2}+\chi_{T}^{2}(4 \text { terms })
$$

where $\chi_{D A T A}^{2}$ and $\chi_{P W}^{2}$ refer to the contributions from data and the existing partial wave solution respectively. The convergence and smoothing are taken care by a convergence test function [1]

$$
\chi_{T}^{2}=\lambda \sum_{n=0}^{N}\left(c_{n}^{+}\right)^{2}(n+1)^{3},
$$

which is added to the $\chi^{2}$ expression with similar terms for the $C^{-}$and $B^{ \pm}$amplitudes. The convergence test function reduces the truncation error in the expansion given in Eq.(2.1) by damping down the coefficients $c_{n}^{+}$for large $n$. The expansion techniques has the additional advantage that data with error bars can be used as input and the error analysis becomes a realistic possibility.

Using the expansion techniques the Karlsruhe group has performed a thorough analysis of pion-nucleon scattering [1]. The analysis has been performed in three stages, the fixed- $t$ analysis, a fixed centre-of-mass analysis and a phase-shift analysis, which were performed iteratively until the amplitudes agree to about $3 \%$. In addition, partial wave dispersion relations [12] and partial wave relations [13] were checked separately and a satisfactory agreement was found.

\section{Recent activity}

The general idea of the Helsinki group has been to make a partial wave analysis of pionnucleon scattering with fixed- $t$ constraints implemented in the same fashion as the Karlsruhe group has done, i.e., with the expansion techniques. The situation in the study of $\pi \mathrm{N}$ interaction has changed considerably from the time when the Karlsruhe analysis was made. Much of the pionnucleon scattering data from the meson factories were published only later and, obviously, the available computer power has increased enormously. The latter fact has made it possible for us to pay more attention to the error analysis.

The focus of our work is at low energies where we wish to make contact to the validity range of chiral perturbation theory and extract some of the low-energy constants.

The analysis of the forward data has been completed and published, see Refs.[14, 15]. The forward dispersion relation for $C^{-}$evaluated at $\omega=\mu$ gives the Goldberger-Miyazawa-Oehme sum rule [16]

$$
C^{-}(\mu)=\frac{8 \pi f^{2}}{\mu\left(1-\left(\frac{\mu}{2 m}\right)^{2}\right)}+4 \pi \mu J^{-}=4 \pi\left(1+\frac{\mu}{m}\right) a_{0+}^{-}
$$




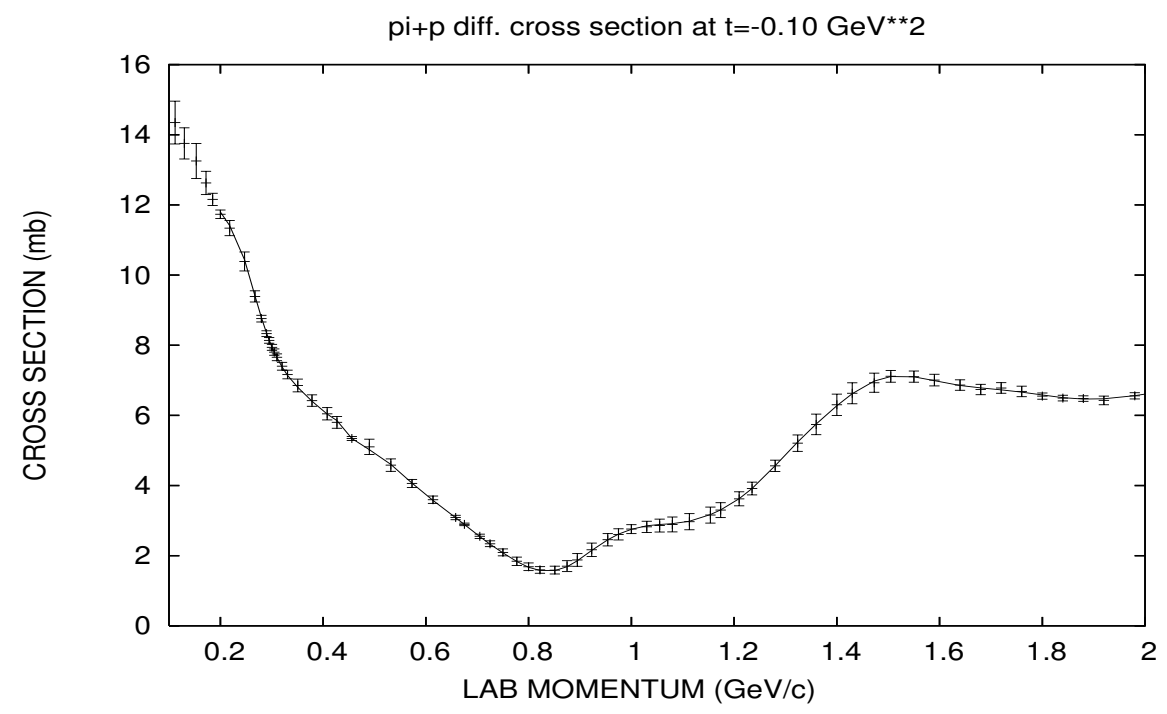

Figure 2: Differential cross section for $\pi^{+} p$ at $t=-0.1 \mathrm{GeV}^{2}$.

where the integral $J^{-}$is defined as

$$
J^{-}=\frac{1}{4 \pi^{2}} \int_{0}^{\infty} \frac{\sigma_{\pi^{-} p}(k)-\sigma_{\pi^{+} p}(k)}{\omega} d k,
$$

$f^{2}$ is the pion-nucleon coupling constant and $a_{0+}^{-}$is the isovector $s$-wave $\pi \mathrm{N}$ scattering length. For $J^{-}$we obtain [14] the value

$$
J^{-}=-1.060 \pm 0.030 \mathrm{mb}
$$

which agrees exatly with the Höhler-Kaiser value of 1980 [17]. They did not, however, perform an error analysis.

If pionic hydrogen $s$-wave level shift information [18] is used together with the correction factor $\delta_{\varepsilon}$ from Ref.[19], the result for the $\pi^{-} p s$-wave scattering length $a_{\pi^{-}}=0.0933 \pm 0.00291 / \mu$ is obtained. Combining this with the value for the $s$-wave $\pi^{+} p$ scattering length [14] $a_{\pi^{+} p}=$ $-0.0764 \pm 0.00141 / \mu$ for the isovector value $a_{0+}^{-}=\left(a_{\pi^{-} p}-a_{\pi^{+} p}\right) / 2$, we get $f^{2}=0.075 \pm 0.002$ from the sum rule. It is clear the the GMO sum rule is not the most accurate method for determining the value for the pion-nucleon coupling constant. However, it is the natural first step in a more comprehensive analysis. Also, it provides a transparent connection between the accuracy of the data and the precision of the result.

The partial wave analysis of pion-nucleon scattering will require the analysis to be extended to $t$-values less than 0 . This turns out to be a challenge in the sense that balancing the weights of the different terms in Eq.(2.3) is cumbersome. As an example, in Fig.2, the $\pi^{+} p$ differential cross section is shown at $t=-0.1 \mathrm{GeV}^{2}$. Here the solid line displays the average of two different partial wave solutions, KA84 of Koch [20] and FA02 of Arndt et al. [21]. The error bars reflect the difference between the solutions. It turns out that the experimental points for the same quantity have error bars which are much bigger than the "theoretical ones" and, therefore, it is difficult to make the partial wave solution to move away from the initial values. 


\section{Conclusions}

In general, the Karlruhe analysis [1] produces a consistent set of partial waves. However, in particular at lower energies, new data have changed the picture and, therefore, a new analysis with fixed- $t$ constraints has become a necessity. The VPI-GWU group [21] has in recent years produced solutions, which also incorporate fixed-t dispersion relations, and they agree well with the Karlsruhe results when viewed at a particular $t$-value. However, the results for the coupling constant do differ. The Karlsruhe value is [22] $f^{2}=0.079 \pm 0.001$ and the VPI-GWU value [21] $f^{2}=0.076 \pm 0.001$.

In the present analysis, until now, the data points have been moved to the pre-selected $t$-values with an existing partial wave solution. Apparently that will not be sufficient, but a more careful data amalgamation will be needed.

\section{Acknowledgments}

This work has been supported in part by the EU Contract MRTN-CT-2006-035482, FLAVIAnet and the European Community Research Infrastructure Integrating Activity HadronPhysics2, Grant Agreement 227431, under the Seventh Framework Programme.

\section{References}

[1] G. Höhler, Landolt-Börnstein, Vol. 9b2, Springer, Berlin 1983.

[2] G. Sommer, Present State of Rigorous Analytic Properties of Scattering Amplitudes, Fortsch. Phys. 18 (1970) 577.

[3] A. Martin, Scattering Theory: Unitarity, Analyticity and Crossing, Lect. Notes Phys. 3 (1969) 1.

[4] R. Oehme, Dispersion relations in quantum chromodynamics, $\pi N$ Newsletter 7 (1992) 1.

[5] R. Oehme, Analytic Structure of Amplitudes in Gauge Theories with Confinement, Int. J. Mod. Phys. A10 (1995) 1995 [hep-th/ 9412040$].$

[6] B. Tromborg, S. Waldenstrøm and I. Øverbø, Electromagnetic corrections to $\pi N$ scattering, Phys. Rev. D15 (1977) 725.

[7] N. Fettes and U.-G. Meißner, Complete analysis of pion-nucleon scattering in chiral perturbation theory to third order, Nucl. Phys. A693 (2001) 693 [hep-ph / 0101030$].$

[8] V.V. Abaev and S.P. Kruglov, Phase-shift analysis of $\pi p$ scattering in the energy region from 160 to $600 \mathrm{MeV}$, Z. Phys. A352 (1995) 85.

[9] D.V. Bugg, $\pi N$ Phase Shifts, 0 - $310 \mathrm{MeV}, \pi N$ Newsletter 6 (1992) 7.

[10] E. Pietarinen, Dispersion Relations and Experimental Data, Nuovo Cim. 12A (1972) 522.

[11] E. Pietarinen, Fixed momentum transfer analysis of pion-nucleon scattering, Nucl. Phys. B107 (1976) 21.

[12] R. Koch and M. Hutt, A Partial Wave Dispersion Relation Analysis of Pion-Nucleon Scattering Amplitudes, Z. Phys. C19 (1983) 119. 
[13] R. Koch, A calculation of low-energy $\pi N$ partial waves based on fixed-t analyticity, Nucl. Phys. A448 (1986) 707.

[14] V.V. Abaev, P. Metsä and M.E. Sainio, The Goldberger-Miyazawa-Oehme sum rule revisited, Eur. Phys. J. A32 (2007) 321, arXiv: 0704.3167 [hep-ph] .

[15] P. Metsä, Forward analysis of $\pi N$ scattering with an expansion method, Eur. Phys. J. A33 (2007) 349 , arXiv:0705.4528[hep-ph].

[16] M.L. Goldberger, H. Miyazawa and R. Oehme, Application of Dispersion Relations to Pion-Nucleon Scattering, Phys. Rev. 99 (1955) 986.

[17] G. Höhler and F. Kaiser, Review and Tables of Pion-Nucleon Forward Amplitudes, Karlsruhe report KfK-3027 (1980) 1.

[18] L.M. Simons, Pionic hydrogen, Int. J. Mod. Phys. A20 (2005) 1644.

[19] J. Gasser et al., Ground-state energy of pionic hydrogen to one loop, Eur. Phys. J. C26 (2002) 13 [hep-ph/0206068].

[20] R. Koch, Improved $\pi N$ Partial Waves, Consistent with Analyticity and Unitarity, Z. Phys. C29 (1985) 597.

[21] R.A. Arndt et al., Dispersion relation constrained partial wave analysis of $\pi N$ elastic and $\pi N \rightarrow \eta N$ scattering data: The baryon spectrum, Phys. Rev. C69 (2004) 035213.

[22] R. Koch and E. Pietarinen, Low-energy $\pi N$ partial wave analysis, Nucl. Phys. A336 (1980) 331. 